\title{
Play-based interventions to support social and communication development in autistic children aged 2-8 years: A scoping review
}

\author{
Jenny L. Gibson ${ }^{1}$ Emma Pritchard ${ }^{1}$ Carmen de Lemos ${ }^{1}$
}

\begin{abstract}
${ }^{1}$ Play \& Communication Lab, Play in Education Development and Learning Research Centre, Faculty of Education, University of Cambridge
\end{abstract}

NB This pre-print has not yet been through peer review. It has been submitted for peer review at the journal Autism and Developmental Language Impairments.

\begin{abstract}
Play is often considered by practitioners to be a natural and enjoyable context for providing intervention and support to autistic children. However, the literature on play-based interventions is large and heterogeneous. This means it is challenging for families and practitioners to navigate the evidence base and make choices about differing play-based approaches. This review aims to provide a comprehensive map of the research on this topic and to develop a conceptual framework to inform decision-making.
\end{abstract}

We used a scoping review methodology to find peerreviewed intervention studies of play-based approaches to targeting language, social and communication outcomes for autistic children aged 2-8 years. We then summarised the literature using narrative synthesis and Evidence Gap Maps (EGMs).

We identified 388 studies, covering many intervention types, for inclusion. Just over $\mathbf{5 0 \%}$ had $<10$ participants. Girls and minority background groups were under-represented. In our summary we suggest important dimensions for appraisal of play-based interventions, including considering the role of play in an intervention and the extent to which approaches are child-led. The wide range of approaches uncovered by this review shows the wonderful diversity of both play and autism. Future research could usefully focus on consolidating the evidence base for existing approaches.

Index Terms-Autism; Play; Intervention; Language; Communication; Social Development

\section{INTRODUCTION}

There is increasing research and clinical interest in the role of play in children's linguistic and social development (Gibson et al., 2020; O’Connor \& Stagnitti, 2011; Stagnitti et al., 2016; Toseeb et al., 2020). For typically developing (TD) children, it is well-documented that early pretend play appears around the end of first year of life, coinciding with the emergence of first words (Quinn et al., 2018; Toth et al., 2006). Other aspects of playful interactions have also been related to broader social competences known to support communicative development, such as joint attention, reciprocal initiations, imitation, and, the giving and reading of nonverbal cues (Godin et al., 2019a; Radley et al., 2014).

Play is often characterised as activities or behaviours that are non-literal, voluntary and fun or rewarding (Miller, 2017). Play takes on a wide range of forms, such as pretence, physical play, sports and digital play and has also been conceptualised as a dispositional trait or stage, e.g. 'playfulness' (Bundy, 2012; Fink et al., 2020; Skaard \& Bundy, 2008) or 'playful engagement' (Godin et al., 2019a). Children with autism spectrum disorder (ASD) show broad differences in the frequency and nature of play behaviours compared to children with typical development (TD) or with other cognitive or developmental conditions (Boutot et al., 2005; Francis et al., 2019). Autistic children's play may be characterised by the presence of repetitive behaviours (Wing et al., 1977) and they may show qualitative differences in imaginative or pretend play relative to other children (Jarrold, 2003). Furthermore, as social and communication difficulties form part of the core diagnostic criteria for ASD (American Psychiatric Association, 2013), it is not surprising that differences in social play have been documented in this population and have been used to inform diagnostic assessments (Gibson et al., 2011; Lord et al., 2000).

The social and communication difficulties experienced by autistic children are associated with a range of negative outcomes including various aspects of daily or adaptive functioning and mood disorder symptomatology (Chang et al., 2012; Rai et al., 2018). Long-term impacts of these difficulties include poorer adaptive and social functioning (e.g. friendships (Magiati et al., 2014) and depression into adulthood (Rai et al., 2018)). Therefore, interventions targeting the development of such skills are considered a priority by autistic individuals and their families (Autistica, n.d.)

The significance of play in the development of communication and social skills, coupled with its inherent 'enjoyableness' as a mode of learning in childhood, has inspired practitioners to develop a range of play-based interventions to foster these skills in autistic children. The research on these play-based interventions, however, varies greatly, including differences in under-pinning philosophies, modes of delivery and conceptualisations of play, as well as in the designs used to assess their effectiveness. Some approaches take a more instrumental approach, e.g. using a game as a good vehicle to learn and practice pragmatic communication skills (Murphy et al., 2019), while others emphasise following a child's voluntary play interests. 
The extent of heterogeneity makes navigating this burgeoning field a challenge from many perspectives, from that of the clinician or family wanting to choose an intervention that is right for a particular child, to the researcher hoping to learn more about the nature of the relations between play and communication in autistic children. The present scoping review aims to address this issue by providing an accessible and comprehensive overview of research on this topic and suggesting new conceptual dimensions that could be used in future appraisal of play-based interventions that target social and communication outcomes.

\section{Reviews of play-based interventions}

Before outlining the methodology used in the current review, we highlight some relevant reviews identified during our preliminary literature searches. Broadly, reviews of play-based interventions for improving social communication skills in autism can be divided into those that focus on improving social play skills in their own right, and those that use playful methods to target other social outcomes.

Kuhaneck and colleagues (Kuhaneck et al., 2020) conducted a systematic review of occupational therapy interventions and examined which therapeutic strategies were more effective in improving play skills. The authors found evidence supporting a range of strategies including environmental modification and adult-led imitation. Although the authors acknowledge the variety of play behaviours and meaning, the approach is mainly focused on play skills to improve in-line with typical developmental expectations. In a similar vein, Kent and colleagues (Kent et al., 2020) reviewed interventions related to play skills. In their systematic review, play-based interventions were included if they met crucial elements of intrinsic motivation, internal control, and the freedom to suspend reality alongside an element of playfulness (Bundy, 2012). Using these criteria, 11 randomised controlled trials (RCTs) were identified. Other reviews in this area investigate the type of social play skills targeted (Kossyvaki \& Papoudi, 2016), playful (Godin et al., 2019b), and interventions that have involved peer-mediated approaches (Lory et al., 2018).

Reviews investigating non-play outcomes relating to social and communication development have focused on specific therapeutic approaches. For example, Lindsey et al. investigated the evidence for LEGO Therapy, while Tiede \& Walton investigated Naturalistic Developmental Behavioral Interventions (NDBIs; (Tiede \& Walton, 2019)).

Overall, the existing reviews we identified are beneficial to understanding particular methods, or types of play-based interventions that have been researched. This body of literature demonstrates some promising findings but also raises important challenges.

Firstly, as discussed by Kent et al. (2019), the definitions of play used across literature are disparate, making synthesis a challenging task. Furthermore, the operationalisation of play within an intervention setting varies widely according to the philosophy or approach underpinning different intervention types. The latter is not always acknowledged by researchers or clinicians but preliminary Patient and Public Involvement
(PPI) consultation for the present study indicated that this aspect is important to autistic individuals and their families. Related to this, we identified no existing review in the literature that could help parents and practitioners to identify and appraise the relative merits of different playbased interventions that target similar outcomes. (sandbank)

The present study

The present study has the following aims

1. To provide a comprehensive map of the research regarding play-based interventions targeting social and communication outcomes for autistic children

2. To develop a conceptual framework for the appraisal of play-based interventions targeting social and communication outcomes for autistic children

\section{Methods}

\section{Approach}

We adopted a scoping review methodology (Munn et al., 2018) The study design was informed by Joanna Briggs Institute guidelines (JBI, (Peters et al., 2015), and the Preferred Reporting Items for Systematic Reviews and MetaAnalysis Extension for Scoping Reviews (PRISMA-ScR) statement (Tricco et al., 2018).

The protocol for the review was registered prospectively on the Open Science Framework (https:/osf.io/ajp29), and updates, changes to this protocol (and the reasons for them) are recorded here)

\section{Community involvement}

We held informal conversations with stakeholders, including autistic adults and parents and practitioners supporting autistic children, to learn more about their views on play and play-based interventions. All agreed on the importance of the topic and the need for clarification about how play can be used to support communication skills, however, conversations revealed varying personal perspectives. Some practitioners felt play was a less appropriate word as children got older, while others thought the emphasis should be on freely chosen, pleasurable activities, kept distinct from 'work' or 'intervention'. Practitioners working in education noted different approaches between mainstream and specialist provision. From an autistic perspective, play was referred to as an escape that helps manage anxiety and promote communication and friendships with people with similar interests. However, autistic adults also reported that play can sometimes cause anxiety and expressed discomfort with the idea that some interventions could be rooted in a behaviourist approach.

This consultation informed our approach to evidence synthesis, as we reflected concerns of stakeholders in our framework for understanding play-based interventions. We also set up a separate, qualitative study to more systematically explore the different perspectives raised (not reported in the present paper). 


\section{Eligibility criteria}

To keep the scope of the review broad yet manageable, we investigated peer-reviewed research with children up to the age of 8 years and concentrated on studies using quantitative or mixed-methods designs. Resources did not permit a greyliterature search. We adopted the Skaard \& Bundy, (2008) characteristics of play to inform judgements about whether an intervention could be considered play-based - intrinsic motivation, internal control and freedom to suspend reality. As such, pure forms of discrete trial teaching (DTT) were not included, even where a toy was offered as a 'reinforcer' as this was not considered an intrinsically motivated, volitional play opportunity.

\section{Sample}

We included studies meeting the following eligibility criteria:

- Children aged 2:0-7:11 years with an autism diagnosis (e.g. autism spectrum disorder, autistic disorder, Asperger's syndrome or pervasive developmental disorder not-otherwise specified (PDD-NOS). Studies of participants with autism + co-occurring conditions were included.

\section{Intervention}

- Study reports a play-based intervention. This criterion can be met when play is explicitly mentioned, or if raters judge that the intervention supports, intrinsic motivation, internal control, and freedom to suspend reality

\section{Outcomes}

- Study reports outcomes regarding social and communication skills, including related skills in social cognition

- Study reports outcome measures that are primarily quantitative in nature; intervention

- Other characteristics

- Study presents original research (including published protocols)

- Published in a peer-reviewed journal

- Studies published since 2009

- Written or translated into English or German

\section{Exclusion characteristics}

- interventions that are too broad in nature to permit 'isolation' of elements relating to play

- interventions solely based on animal-assisted therapies, DTT, cognitive behavioural therapy, social media, or social stories.

- outcomes solely related to educational achievement, sensory experience, routines, special interests, and inflexibility

- outcome measures that are entirely qualitative

- Reviews, and meta-analyses

- book chapters
Search strategy

Terms relating to autism, play-based intervention, social/communication outcomes, and those implying an intervention study were combined for the literature search (see appended example, or online protocol for full strategy). A combination of free text (across title/abstract/keyword fields) and subject heading terms were used, and the search strategy was adapted for each database.

Electronic searches using these terms were conducted on 7 and 8 November 2019 across seven electronic databases covering the period 01/01/2000 -06/11/2019: British Education Index (BEI), Child Development \& Adolescent Studies, ERIC, PsycINFO, PubMed, Scopus and Web of Science Core Collection. Filtering by publication date, participant age and publication or source type (those published in academic journals), was carried out after searching. There were no restrictions by language.

For quality assurance, the Web of Science and PsycINFO searches were peer-reviewed by a research librarian using the Peer Review of Electronic Search Strategies (PRESS) checklist (McGowan et al., 2016).

The electronic database searching was supplemented by scanning the reference lists of selected relevant reviews. Authors of potentially relevant but inaccessible articles were contacted up to three times to request full text articles. Articles that remained inaccessible were excluded.

\section{Study selection}

The search yielded 18,645 papers, which we uploaded into EPPI-Reviewer 4 software (Thomas et al., 2010). Handsearching techniques yielded 7 further studies. Figure 1 shows the PRISMA-ScR process diagram of study selection within this review.

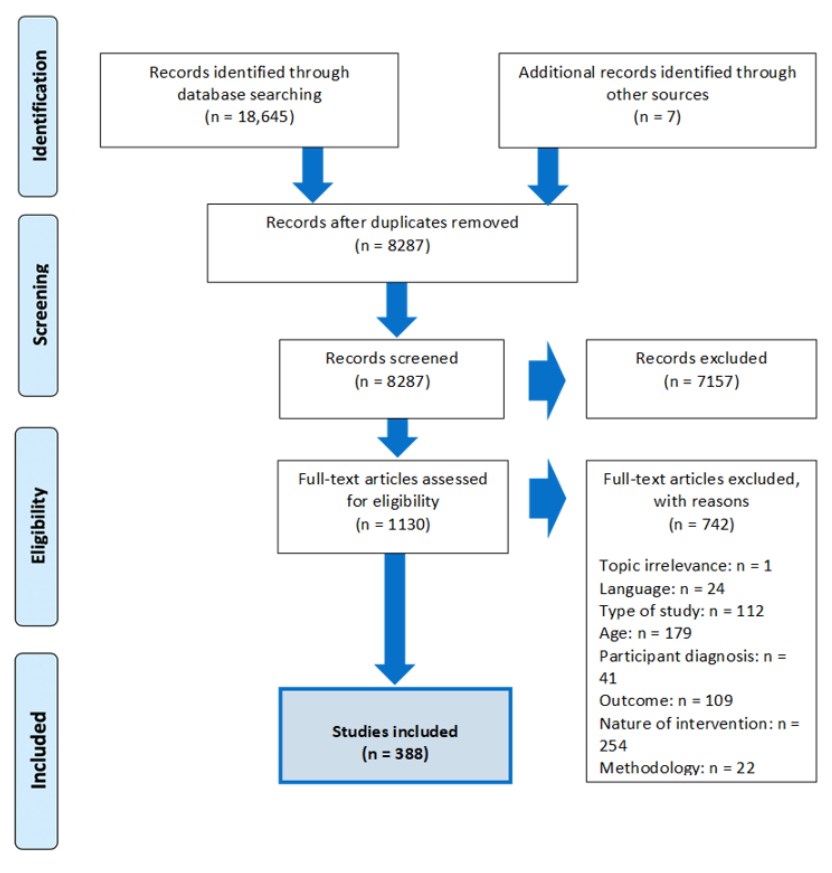

Figure 1. Study selection flow diagram 
After deduplication, 8,287 studies were screened for relevance based on title and abstract, $3 \%$ of these (248) were independently double-coded for inclusion, percentage agreement $=93.98 \%$, Cohen's kappa $(x)=.818(95 \%$ CI, .646 to $.990), \mathrm{p}<.001$. Any disagreements were resolved through discussion.

The remaining 1,130 articles were full-text reviewed against the study inclusion and exclusion criteria. $20 \%$ of these were double-blinded coded; $81 \%$ agreement and moderate $x=.619$ (95\% CI,.466 to .772), $\mathrm{p}<.001$.

\section{Charting and synthesising the data}

The data charting process is the first step in summarising and synthesising across the evidence base. Aside from the codes based on the inclusion and exclusion criteria, we wished to chart important commonalities across the different studies. Therefore, creation of charting codes was an iterative process involving discussion and reflection between all authors, guided by principles set out by Gough, Oliver \& Thomas (Gough et al., 2017; Thomas et al., 2017). The full data charting codes and categories are in the supplementary materials (S1).

The following data were recorded for each included study: Bibliographic data; data relevant to inclusion criteria; participant information; intervention details; categories of play; outcome domain; agent of intervention. Classification under these different headings was double-blind coded for $20 \%$ of the 388 included studies, average $x=.75$ across all codes.

Once charting was complete, we then conducted a narrative synthesis to address the second aim of developing a conceptual framework that can help practitioners and others navigate and appraise the extensive literature in this area.

Managing the high number of studies

After full-text review, 388 studies met the inclusion criteria (See Figure 1, plus list of included studies in supplementary materials (S2)). As this number of studies is too large to tabulate in the context of an academic paper, we took a different approach to that of scoping studies with smaller samples. We provide the full list of studies online using an interactive evidence gap map (EGM). This allows the user to summarise charted characteristics across important dimensions, according to their own interests. In the results section we provide quantitative summaries of this evidence under different headings. Secondly, given our aim to provide practitioners with a useful way to navigate the evidence base, we selected a subset of interventions meeting the following criteria:

1) Intervention should be the subject of at least two research studies, and

2) be a named or 'manualised' intervention approach

We refer to this subset of studies as 'named intervention' studies and they are tabulated in supplementary materials (S3).

The narrative synthesis element can be found in the 'discussion' section of the present paper.
Deviations from protocol

Deviations from the original protocol were largely the result of pragmatic decisions to enable the work to fit with available resources, however some changes were made on the basis of reflection, consultation with stakeholders and findings of unexpected complexity in some areas where our original thoughts no longer applied. All changes from protocol are documented here.

\section{Results}

A total of 388 studies met inclusion criteria. The full list of included studies is in the supplementary materials. Below we report results of the charting and synthesis process, starting with our approach to mapping different types of play-based interventions and moving on to considering the characteristics of the evidence-base.

\section{Play-based intervention types}

To meet our first aim of providing a comprehensive map of the research regarding play-based interventions targeting social and communication outcomes, we devised categories to group together play-based interventions with a common methodological approach or aim, see Table 1 . The categories are not necessarily mutually exclusive although for each study in the review we have attempted to identify the main intervention type wherever possible.

\section{The role of play within interventions}

We also characterised the interventions along two main dimensions relating to their conceptualisation of play:

1) "role of play" - the extent that playful engagement is considered the key mechanism of developmental change, and

2) "level of child control" - the extent to which the intervention is child-led

Codes relating to these aspects of play are shown in Table 2 , and the frequency of each aspect of play by intervention type is shown in Figures 2 and 3.

\section{Social communication outcomes}

The present review focuses on interventions targeting outcomes related to social and communication development. To summarise the vast array of potential intervention targets in these areas, we devised 8 broad outcome categories that capture the main area of focus for each study (see supplemental materials S4).

Figure 4 shows the proportion of studies $(\mathrm{N}=388)$ for each outcome domain. The most common category is the 'multiple' outcomes category, with over $45 \%$ of studies using more than one relevant outcome. Social play skills are the next most common target of play-based interventions. 
Table I

INTERVENTION TYPES

\begin{tabular}{|c|c|}
\hline $\begin{array}{l}\text { Intervention } \\
\text { type }\end{array}$ & Description \\
\hline $\begin{array}{l}\text { Feedback- } \\
\text { based }\end{array}$ & $\begin{array}{l}\text { Practitioner provides tailored feedback to the target child and/or their } \\
\text { interaction partner during or after play. The play partner may be a } \\
\text { parent, other professional, or a peer. }\end{array}$ \\
\hline $\begin{array}{l}\text { Influenced by } \\
\text { behaviourist } \\
\text { approach }\end{array}$ & $\begin{array}{l}\text { Judged as having been influenced by Applied Behaviour Analysis } \\
\text { (ABA) principles such as discrete trial teaching and positive } \\
\text { reinforcement, to varying degrees. May range from traditional to more } \\
\text { developmental interpretations of ABA. }\end{array}$ \\
\hline $\begin{array}{l}\text { Computer- } \\
\text { based }\end{array}$ & Delivered via computer (including video games). \\
\hline Robot-assisted & Delivered via robot technologies. \\
\hline Virtual Reality & Involve the use of virtual reality games. \\
\hline $\begin{array}{l}\text { Augmentative } \\
\text { and alternative } \\
\text { communication } \\
\text { (AAC) }\end{array}$ & Low- or high-tech technologies to support successful communication. \\
\hline $\begin{array}{l}\text { Video } \\
\text { modelling }\end{array}$ & $\begin{array}{l}\text { Pre-made or individualised videos consisting of modelled targets. The } \\
\text { child watches these and imitates the modelled target behaviours with } \\
\text { varying support in appropriate settings. }\end{array}$ \\
\hline $\begin{array}{l}\text { Early intensive } \\
\text { intervention }\end{array}$ & $\begin{array}{l}\text { Comprehensive intervention programmes, delivered over a period of } \\
\text { time shortly after a child receives an autism diagnosis. }\end{array}$ \\
\hline $\begin{array}{l}\text { Social skills } \\
\text { group }\end{array}$ & $\begin{array}{l}\text { Delivered to groups of children. They often support the development of } \\
\text { holistic or specific social and communication skills. }\end{array}$ \\
\hline $\begin{array}{l}\text { Art and } \\
\text { outdoor } \\
\text { activity }\end{array}$ & Activity-based interventions such as aquatic or music therapy. \\
\hline Play therapy & $\begin{array}{l}\text { Typically delivered by specialist play therapists trained in child } \\
\text { psychotherapy. }\end{array}$ \\
\hline $\begin{array}{l}\text { Activity } \\
\text { schedule }\end{array}$ & $\begin{array}{l}\text { Interventions which use a visual breakdown of steps related to a target } \\
\text { activity to support the child's successful or appropriate involvement. }\end{array}$ \\
\hline $\begin{array}{l}\text { Collaboration- } \\
\text { based }\end{array}$ & $\begin{array}{l}\text { Collaborative working with others for a task within an intervention to be } \\
\text { successful. }\end{array}$ \\
\hline Mixed & A mixture of the above intervention types. \\
\hline
\end{tabular}

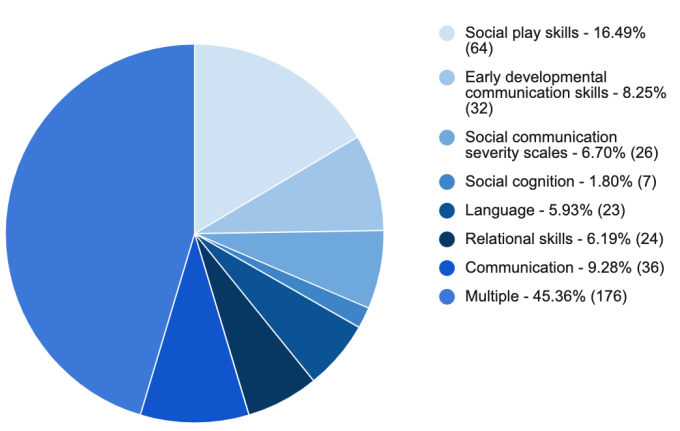

Figure 4. Outcomes targeted by play-based interventions
Agent of intervention (AoI)

Given the scope of interdisciplinary involvement in playbased interventions, we aimed to record those who were actively involved in delivering the intervention in relation to its theorised mechanisms of change. Despite AoI-type codes being a common feature of many intervention-related reviews, this category was difficult to implement in practice for many reasons, including - multiple agency delivery or involvement, unclear reporting, the role of the main agent or others involved being complex and variable practices across interventions/studies. Two over-arching categories were de- 
Table II

AsPeCTS OF PLAY CODES

\begin{tabular}{ll}
\hline Role of play & Description \\
\hline Component & $\begin{array}{l}\text { An aspect of free play or structured game play is included } \\
\text { as part of an intervention session(s), or as part of } \\
\text { a programme that has multiple components. }\end{array}$ \\
Context & $\begin{array}{l}\text { Play is conceptualised as a context for intervention. For } \\
\text { example, it could target social engagement set in an } \\
\text { environment conducive to play, such as a playground or } \\
\text { play corner in a nursery. }\end{array}$ \\
Key mechanism & $\begin{array}{l}\text { The intervention actively uses play within the intervention } \\
\text { as a key mechanism for change. }\end{array}$ \\
\hline Level of child-control & $\begin{array}{l}\text { Highly structured, the adult is in control determines the } \\
\text { target and type of play. }\end{array}$ \\
Adult-led intervention & $\begin{array}{l}\text { A structured intervention that allows the child freedom } \\
\text { within the limits of that structure, or a combination of } \\
\text { adult-led and child-led play is used. } \\
\text { An intervention which allows the child to engage in free } \\
\text { play or follows the child's lead, for example using } \\
\text { techniques to expand upon utterances or prolong shared } \\
\text { attention when something has caught a child's interest. } \\
\text { Insufficient information provided to make a judgement } \\
\text { based on these categories. }\end{array}$ \\
Child-led intervention & $\begin{array}{l}\text { Level of child-control not } \\
\text { clear }\end{array}$ \\
\hline
\end{tabular}

Augmentative and alternative communication

Activity schedule

Video modelling

Play therapy

Art and outdoor activity

Influenced by behaviourist approach

Feedback-based

Collaboration-based

Virtual Reality

Robot-assisted

Computer-based

Early intensive intervention

Social skills group
1

2

11

1

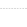

1

3
1

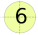

14

12

77

5

3

16

1

6

Key Mechanism
5

Component

Figure 2. Role of play by intervention type 


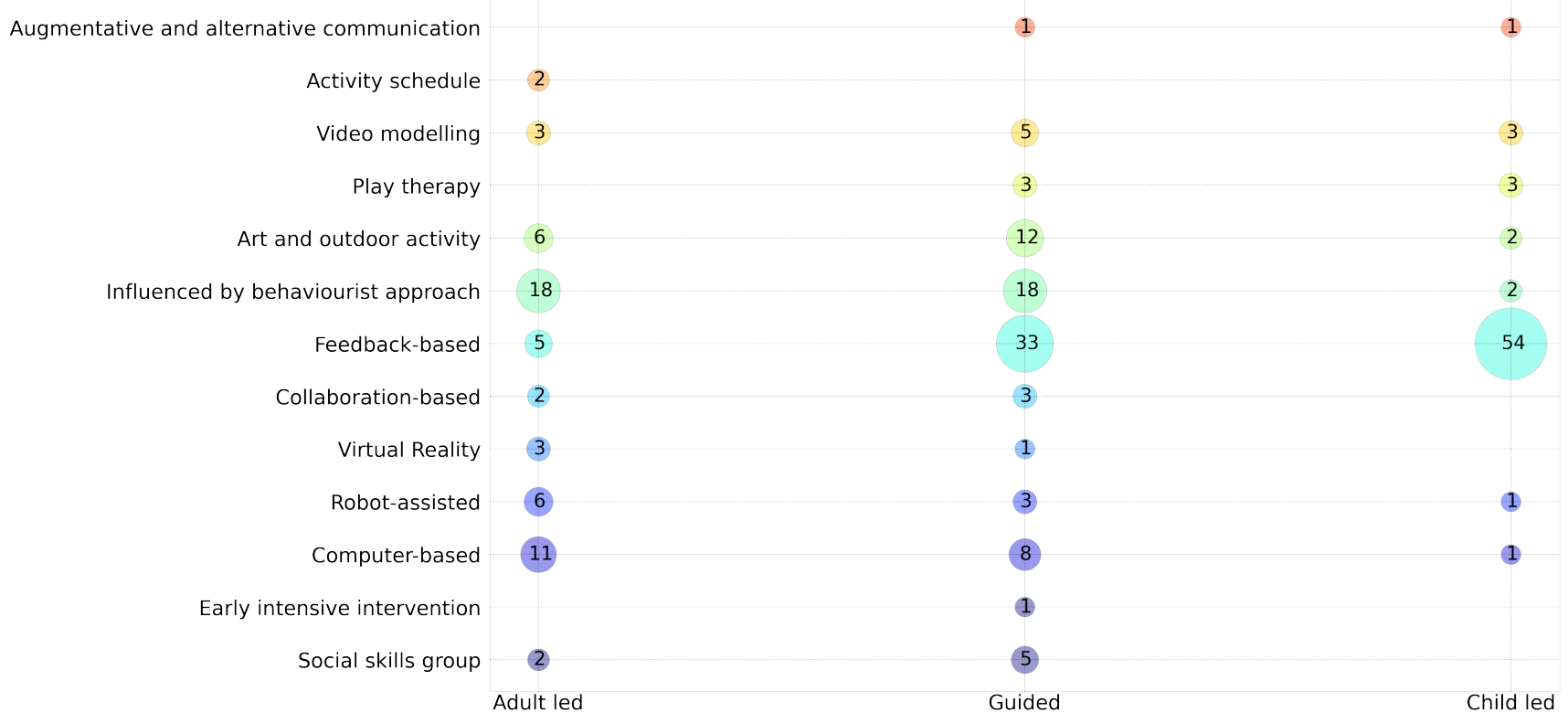

Figure 3. Level of child control

veloped:

1) 'Main agent of intervention' - the background or profession of the main interventionist or clinical researcher leading the delivery of the intervention; or multidisciplinary team (MDT), and

2) 'Others involved' - individuals also actively involved in the intervention delivery who are not considered under the 'Main agent' category. Typically, this concerns studies in which the main agent trains an individual to implement the intervention and includes a variety of non-professionals or paraprofessionals. Note that robots are not considered as part of these categories.

The frequency for each subcategory within the AoI codes is shown in Table 3.

Table III

Agent of INTERVENTION FREQUENCIES

\begin{tabular}{lc}
\hline AoI category & Count \\
\hline Main agent & \\
\cline { 1 - 2 } ABA/Behaviourist training or background & 15 \\
Arts/creative therapies & 8 \\
Education & 47 \\
Mixed professions/ interdisciplinary teams & 74 \\
Non-specified & 147 \\
Occupational Therapy & 4 \\
Other & 9 \\
Play Therapy & 2 \\
Psychology & 53 \\
Speech \& Language Therapy & 29 \\
\hline Others involved & \\
\hline None & 179 \\
Other & 3 \\
Parent/Carer & 136 \\
Peer & 32 \\
Sibling & 6 \\
Teacher & 31 \\
Teaching assistant/paraprofessional & 25 \\
\hline
\end{tabular}

\section{Participant characteristics}

Three hundred and sixty-four studies (94.79\%) included an autistic-only sample and 52 studies (13.54\%) included autistic participants with co-occurring conditions. Sixty percent studied females and males, although mixed samples tended to include more males. Just $1.6 \%$ of studies looked at playbased interventions for girls only and $28.9 \%$ investigated boys only. The remaining studies did not report the gender/sex of their participants.

Reports of participant IQ and adaptive functioning were found in a substantial minority of studies. Figure 5 shows the breakdown for the 132 studies reporting IQ and Figure 6 shows comparable data for the Vineland Adaptive Behavior Scale (VABS) adaptive functioning scores. Table 4 shows the numbers of studies reporting these and other relevant characteristics such as language ability or autistic symptomatology scores.

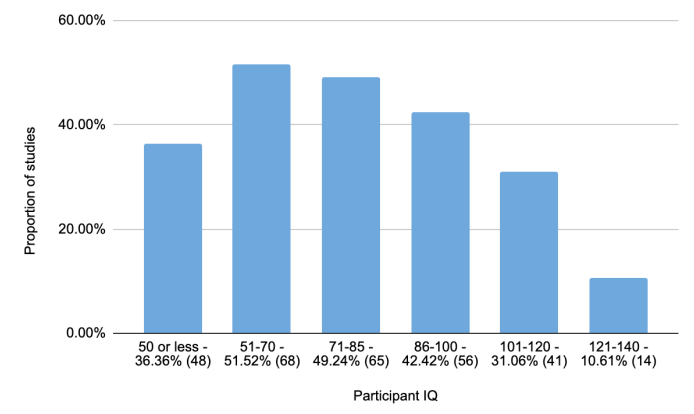

Figure 5. IQ range 
Table IV

FUNCTIONAL INDICATORS

\begin{tabular}{ll}
\hline Functional indicator & $\%(\mathrm{n})$ \\
\hline IQ score & $34.02(132)$ \\
Adaptive functioning (VABS) score & $31.96(124)$ \\
Functional description & $43.56(169)$ \\
ASD symptomatology diagnostic score & $40.21(156)$ \\
Language measure & $23.71(92)$ \\
Developmental or mental age equivalent & $15.98(62)$ \\
Not given & $8.25(32)$ \\
\hline
\end{tabular}

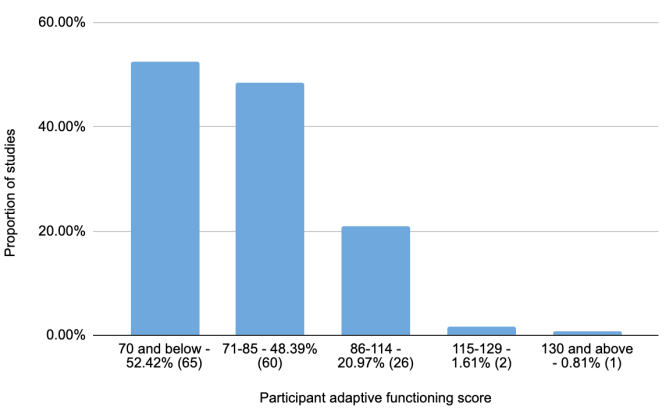

Figure 6. Adaptive functioning range

Moving to consider demographic and contextual characteristics, a substantial majority of the research has been produced in the USA, followed by Australia, Canada and UK. Most studies did not provide information about ethnicity $(\mathrm{n}=239,61.6 \%)$, family income $(\mathrm{n}=349,89.9 \%)$, parental occupation $(n=364,93.8 \%)$, or maternal education levels $(n=287$, $74 \%$ ). Where reported, the most common ethnicity categories were White, Latinx/Hispanic, Black, Asian and Mixed/Other. Just five studies reported on indigenous or first nation cultures in the regions where the highest number of studies were conducted.

Study design

The coding of study designs was adapted from the three 'level of evidence' ratings used by The Communication Trust's Better Communication Research Project (Law et al., 2012), see Supplementary materials S5. Note that while the study design categories give an indication of the overall quality of the evidence when it comes to intervention effectiveness, such that an RCT is considered more robust evidence than a quasi-experimental study and so on, the 'level of evidence' categorisation does not give any information about the quality or findings of an individual study. The percentage representation of each study design is shown in Figure 7.

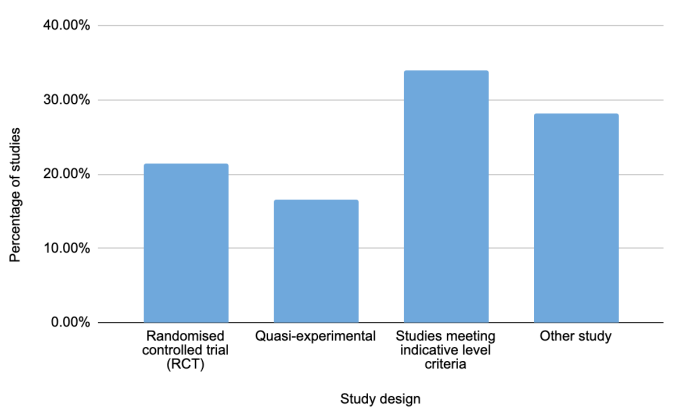

Figure 7. Study designs

Fidelity, generalisation and follow-up

Excluding protocols $(n=4), 53.13 \%$ of studies reported some measure of treatment fidelity, while $28.09 \%(n=109)$ reported a generalisation phase, and $39.69 \%(\mathrm{n}=154)$ reported longer term follow up after the immediate post-intervention measurement, 9 of these studies were separate papers reporting a follow-up study from an earlier intervention, the rest report follow-up in the same paper.

Evidence gap maps

Readers are strongly encouraged to explore the interactive, online evidence gap maps by clicking here. This will allow users to investigate the features of an intervention of interest, for example if it is child-led, guided or adult-led play, or whether or not it is linked to behaviourist methods. The screenshot in Figure 8 below, gives an example:

The small boxes each represent a study and in this case they are coloured according to the child-led code. So in the screenshot we can see that feedback-based interventions targeting social play skills tend to use guided play approaches.

\section{Discussion}

\section{Conceptualising play-based interventions}

The major contribution of the present paper is to summarise the heterogenous literature on the role of play in social and communication interventions in a way that has stakeholder relevance. The conceptualisation of the role of play within such interventions as falling within 3 broad categories of 'context', 'component' or a 'key mechanism' will support practitioners in reflections about their own views and practices regarding play. This approach reflects the theoretical and empirical play literature that asks questions about exactly how play activities or playful engagement might promote child development (Gibson et al., 2020; Godin et al., 2019a; Hopkins et al., 2015; Luckett et al., 2007), as well as the literature that emphasises environmental adaptation, or 'context-based' therapies (Bundy et al., 2009, 2016).

Likewise, the 'level of child control' categories resonate with the literature on developmentalist strategies for intervention and debates around the role of guided-play, vs direct instruction (Weisberg et al., 2016). The present review found that feedback-based approaches most frequently employ play as a key mechanism and are likely to be childled. Interestingly, technology-based approaches tended to be 


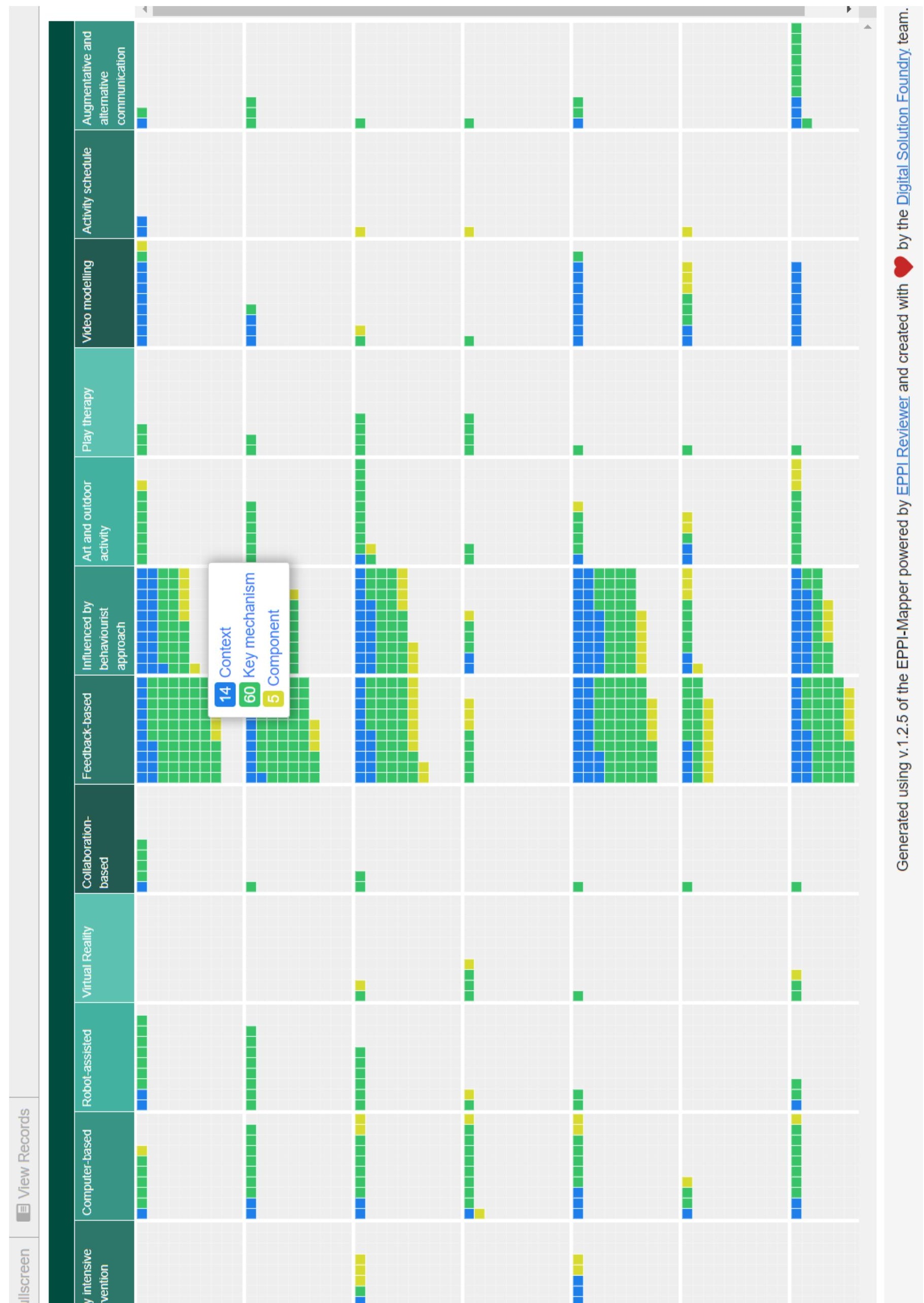


more adult-directed, perhaps requiring a greater level of adult control to facilitate their use. More naturalistic approaches to technology use in autism have shown that free-play may be associated with higher levels of social and co-operative behaviour (Francis et al., 2019), so it is interesting to note that such approaches have not yet translated into intervention research.

The stakeholder consultation was also helpful in informing our approach to developing these two dimensions for scoping the literature. We found that play-based interventions were generally acceptable to practitioners, autistic people and the families of autistic people, although some concerns were raised from autistic individuals about the need to think carefully regarding bringing special interests into therapies and to consider the importance of autonomy. This chimes with Fletcher-Watson's finding of a positive view of early intervention support (Fletcher-Watson et al., 2016). We hope that by reflecting on the extent to which their intervention practices are child-led and the different ways in which play underpins an intervention approach, practitioners can help autistic children and their families make informed choices.

\section{Types of play-based interventions}

Categories for intervention types were developed reflecting the main intervention approaches that we judged would be of clinical interest. Furthermore, as some autistic stakeholders mentioned that they would be concerned about play-based therapies masking an underlying Applied Behaviour Analysis (ABA) approach, and some parents of autistic children wanted more information about ABA therapies, we also factored in information about the underlying philosophy, where this could be discerned. It is worth noting that this is not always self-evident, for example in the broader literature parent feedback models have been developed from both operant conditioning (Brookman-Frazee et al., 2006) and attachment theory perspectives (Juffer et al., 2017). In the present review, feedback approaches were one of the most common intervention types and were largely based on more naturalistic behaviourist approaches (e.g. ESDM), or neuroconstructivist models (e.g. PACT). Although feedback-based approaches were mainly based on parenting interventions, there is also an emerging literature concerning peer feedback, including friends and siblings.

We also identified intervention types that are more likely to be carried out by practitioners based in schools, such as social skills groups, interest-based activity groups (e.g. music, drama) or collaborative play (e.g. LEGO-based therapy). These reflect the increasingly important role of peers in children's learning and development as they move into the primary school years (Howe, 2009), and a move away from seeing play as a key developmental mechanism toward more of a learning context as children get older. We did not find any play-based interventions targeting social and communication outcomes that were explicitly strengths-based, or which view peer-peer interventions between autistic individuals as a deliberate strategy for supporting autistic communication styles (as contrasted with incidental groupings, e.g. due to school placement). This could be an interesting avenue for future play interventions, given recent findings of enhanced communication between autistic/autistic pairs, compared to autistic/non-autistic pairs (Crompton et al., 2020) and calls for strengths-based approaches from autism research organisations internationally (Huntley et al., 2019) .

Having discussed the broader parameters used to scope the literature, we turn to discussion of more specific aspects of the studies reviewed, beginning with study design.

\section{Study design}

Just $20 \%$ of the included studies used an RCT design, which is typically considered the most robust way to test intervention efficacy (Greenhalgh, 2014). Quasi-experimental designs, using a control group but with no random allocation, accounted for a further $17 \%$, meaning most included studies used designs that cannot provide conclusive or convincing evidence of clinical efficacy. Thus, we can conclude that the current evidence base is limited, even though we did not specifically evaluate effect-sizes/outcomes.

The lack of robust intervention studies may be because play-based interventions are a relatively new type of 'complex intervention' requiring extensive, iterative development and testing before they are worth the expense of a large trial and can be effectively taken to scale (O'Cathain et al., 2019). Choosing a study design that fits the state of knowledge in the discipline and addresses ethical or pragmatic concerns is entirely appropriate in a field in its early stages. Robotbased and computer-based interventions are good examples of approaches at an early stage. Papers classed as 'other' or 'indicative' for these types of interventions often include discussion of the development process and stakeholder consultation, important features of inclusive research (FletcherWatson et al 2019).

On the other hand, it is concerning that more than half of the included studies had 10 or fewer participants, and $43 \%$ had fewer than 5 participants. While case-studies, caseseries and small-scale observational work can provide an important foundation for intervention research, the field needs to consolidate and scale-up research efforts if widely implementable evidence-based interventions are the ultimate ambition.

\section{Participants}

Reporting of participant characteristics was piecemeal. While all studies included autistic participants, just $13 \%$ report co-occurring conditions. This is in contrast with reports of a high frequency co-occurring conditions in the literature. In line with many other autism related studies, girls were also under-represented despite having a potentially different social phenotype (Van Wijngaarden-Cremers et al., 2014).

\section{Agent of intervention and outcomes}

Practitioners from many backgrounds have been creative in finding ways to use play to support autistic children facing challenges in communication and social interaction. We were 
surprised by the relatively low number of OT interventions, given that the field long standing tradition of play-based intervention, and also by the relatively few SaLT studies, given our focus on communication-based outcomes. Overall, the data highlight the importance of multi-disciplinary approaches in this field (Strunk et al., 2017). Congruent with a multi-disciplinary approach, we also found that playbased interventions frequently target multiple outcomes - an approach that makes sense given the pervasive nature of social communication difficulties in autism. Social play skills were the largest single type of outcome target, measured in $16.5 \%$ of studies. This ties in with the increasing recognition that play is a crucial tool for supporting learning and development, particularly in social domains (Toseeb et al., 2020; Weisberg et al., 2013; Whitebread et al., 2009). Language development and early communication skills development were also popular as intervention targets, particularly for studies using Feedback and/or behaviourist influenced approaches.

The distinction between those studies which use play as a medium for interventions targeting other outcomes, and those which target social play skills as an outcome per se, will be useful to consider when making decisions about which play-based interventions to support in future research.

\section{Strengths and Limitations}

This review synthesises evidence from across a wide range of play-based interventions, allowing practitioners to position their own intervention approaches within a broader context and highlighting features of interventions that can inform clinical decision making. Furthermore, use of a preregistered design and independent inter-rater reliability for key codes demonstrates a rigorous approach. Nevertheless, it is important to consider limitations. Firstly, due to resource constraints, we have not been able to search grey literature and therefore could have missed important new approaches. Secondly, we have not included quality appraisals or outcome syntheses, meaning that questions of intervention efficacy cannot be addressed. While this is an omission in-keeping with the decision to use scoping review methods, effectiveness data would be an important next-step for evidence synthesis in this area. Readers could refer to existing metaanalyses on early intervention for some insight into evidence of efficacy (e.g. (Sandbank et al., 2020)). Finally, due to the extent and variation of specific outcome measures used in autism research and clinical practice, we have not reported the exact measurement tools that studies used within these domains. An excellent review of outcome measurement tools can be found in this comprehensive report by McConachie and colleagues (McConachie et al., 2015).

\section{Conclusions and clinical implications}

The wide range of approaches uncovered by this review is a testament to the wonderful diversity inherent to both play and autism. However, future research could usefully focus on consolidating the evidence base for existing play-based interventions, as the overall impression is that evidence is sparse and tending to lack robust methods. The field would also benefit from greater attention to diversity of sampling,

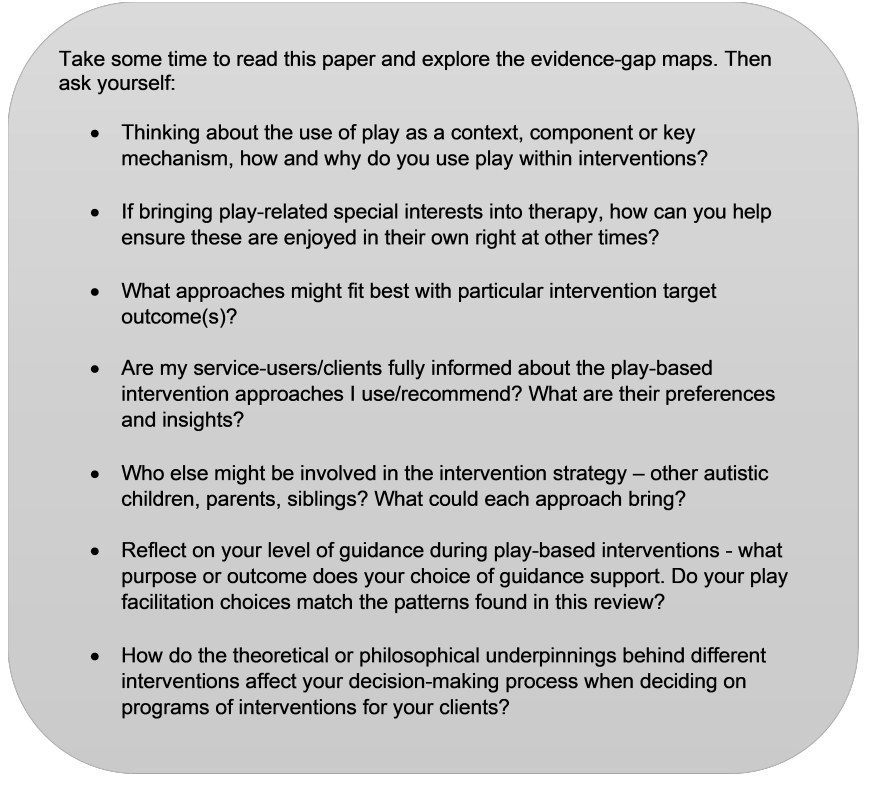

Figure 9. Practitioner reflection and discussion prompts

making sure to include girls and minority background individuals, and to conduct studies in a wider range of countries from more diverse socio-economic contexts.

Regarding clinical implications, the conceptual framework proposed in this review can help practitioners appraise the literature and aid their advice to families when making shared intervention decisions. Figure 9 contains some reflection and discussion prompts for practitioners to support engagement with the findings of this review.

The stakeholder consultation and review findings reveal important dimensions for appraisal of play-based interventions, including the role of play, the underpinning philosophy (e.g. behaviourist or developmental), the role of the practitioner (providing parent feedback, 1:1 intervention, group facilitation), and the involvement of others in implementation of play-based approaches.

Acknowledgements: We gratefully acknowledge funding from LEGO Foundation. We thank Angela Cutts and the team at the Everton Library, and Isla Kuhn and the team at the Medical Library for invaluable support, advice, and peer review. Thanks to the EPPI reviewer team for their support and patience.

Conflict of interest declarations: This study was funded by LEGO Foundation. LEGO therapy was coded in this review.

\section{REFERENCES}

American Psychiatric Association. (2013). Diagnostic and statistical manual of mental disorders: DSM5. American Psychiatric Association. In DSM. https://doi.org/10.1176/appi.books.9780890425596.744053

Autistica. (n.d.). Autistica Research Priorities. Https://Www.Autistica.Org.Uk/OurResearch/Our-Research/Your-Research-Priorities. https://www.autistica.org.uk/our-research/our- 
research/your-research-priorities

Boutot, E. A., Guenther, T., \& Crozier, S. (2005). Let's play: Teaching play skills to young children with autism. Education and Training in Developmental Disabilities, 40(3), 285-292. https://www.scopus.com/inward/record.uri?eid=2-s2.0$26844524329 \&$ partnerID $=40 \& m d 5=342 \mathrm{c} 852 \mathrm{e} 2511 \mathrm{c} 2313275631 \mathrm{c} 4$

Brookman-Frazee, L., Stahmer, A., Baker-Ericzén, M. J., \& Tsai, K. (2006). Parenting interventions for children with autism spectrum and disruptive behavior disorders: Opportunities for cross-fertilization. Clinical Child and Family Psychology Review, 9(3-4), 181-200. https://doi.org/10.1007/s10567-006-0010-4

Bundy, A. (2012). Children at play: Can I play too? In S. J. Lane \& A. C. Bundy (Eds.), Kids can be kids: A childhood occupations approach (pp. 28-43). F.A. Davis.

Bundy, A., Luckett, T., Tranter, P., Naughton, G., Wyver, S., Ragen, J., \& Spies, G. (2016). The Sydney playground project: Results from a cluster randomized controlled trial of a school playground-based intervention to increase children's physical activity, play and social skills.

Bundy, A., Tranter, P., Naughton, G., Wyver, S., \& Luckett, T. (2009). Playfulness: interactions between play contexts and child development (pp. 76-87). Oxford University Press. https://researchers.mq.edu.au/en/publications/playfulnessinteractions-between-play-contexts-and-child-developm

Chang, Y. C., Quan, J., \& Wood, J. J. (2012). Effects of Anxiety Disorder Severity on Social Functioning in Children with Autism Spectrum Disorders. Journal of Developmental and Physical Disabilities, 24(3), 235-245. https://doi.org/10.1007/s10882-012-9268-2

Crompton, C. J., Ropar, D., Evans-Williams, C. V., Flynn, E. G., \& Fletcher-Watson, S. (2020). Autistic peerto-peer information transfer is highly effective. Autism, 136236132091928. https://doi.org/10.1177/1362361320919286

Fink, E., Mareva, S., \& Gibson, J. L. (2020). Dispositional playfulness in young children: A cross-sectional and longitudinal examination of the psychometric properties of a new child self-reported playfulness scale and associations with social behaviour. Infant and Child Development. https://doi.org/10.1002/icd.2181

Fletcher-Watson, S., Apicella, F., Auyeung, B., Beranova, S., Bonnet-brilhault, F., Canal-bedia, R., Charman, T., Chericoni, N., Conceição, I. C., Davies, K., Farroni, T., Gomot, M., Jones, E., Kaale, A., Kapica, K., Kawa, R., Kylliäinen, A., Larsen, K., Lefort-besnard, J., ... Salomone, E. (2016). Attitudes of the autism community to early autism research. Autism. https://doi.org/10.1177/1362361315626577
Francis, G. A., Farr, W., Mareva, S., \& Gibson, J. L. (2019). Do Tangible User Interfaces promote social behaviour during free play? A comparison of autistic and typicallydeveloping children playing with passive and digital construction toys. Research in Autism Spectrum Disorders, 58. https://doi.org/10.1016/j.rasd.2018.08.005

Gibson, J. L., Fink, E., Torres, P. E., Browne, W. V., \& Mareva, S. (2020). Making sense of social pretense: The effect of the dyad, sex, and language ability in a large observational study of children's behaviors in a social pretend play context. Social Development, 29(2), 526-543. https://doi.org/10.1111/sode. 12420

Gibson, J. L., Hussain, J., Holsgrove, S., Adams, C., \& Green, J. (2011). Quantifying peer interactions for research and clinical use: The Manchester Inventory for Playground Observation. Research in Developmental Disabilities, 32(6), $2458-2466$

Godin, J., Freeman, A., \& Rigby, P. (2019a). Conceptual clarification of the playful engagement in social interaction of preschool-aged children with autism spectrum disorder (ASD). Early Child Development and Care, 189(3), 430-440. https://doi.org/10.1080/03004430.2017.1324437

Godin, J., Freeman, A., \& Rigby, P. (2019b). Interventions to promote the playful engagement in social interaction of preschool-aged children with autism spectrum disorder (ASD): a scoping study. Early Child Development and Care, 189(10), 1666-1681. https://doi.org/10.1080/03004430.2017.1404999

Gough, D., Oliver, S., \& Thomas, J. (2017). An introduction to systematic reviews (2nd ed.). Sage Publications Ltd.

Greenhalgh, T. (2014). How to read a paper: the basics of evidence-based medicine (5th ed.). BMJ Books.

Hopkins, E. J., Dore, R. A., \& Lillard, A. S. (2015). Do children learn from pretense? Journal of Experimental Child Psychology. https://doi.org/10.1016/j.jecp.2014.09.004

Howe, C. (2009). Peer Groups and Children's Development. Wiley. https://doi.org/10.1002/9781444318098

Huntley, M., Black, M. H., Jones M, \& Falkmer, M. (2019). Action Briefing: Strengths-based Approaches. https://doi.org/10.1002/aur.1592

Jarrold, C. (2003). A review of research into pretend play in autism. Autism, 7(4), 379-390. https://doi.org/10.1177/1362361303007004004

Juffer, F., Struis, E., Werner, C., \& Bakermans-Kranenburg, M. J. (2017). Effective preventive interventions to support parents of young children: Illustrations from the Videofeedback Intervention to promote Positive Parenting and 
Sensitive Discipline (VIPP-SD). Journal of Prevention \& Intervention in the Community, 45(3), 202-214. https://doi.org/10.1080/10852352.2016.1198128

Kent, C., Cordier, R., Joosten, A., Wilkes-Gillan, S., Bundy, A., \& Speyer, R. (2020). A Systematic Review and Meta-analysis of Interventions to Improve Play Skills in Children with Autism Spectrum Disorder. Review Journal of Autism and Developmental Disorders, 7(1), 91-118. https://doi.org/10.1007/s40489-019-00181-y

Kossyvaki, L., \& Papoudi, D. (2016). A Review of Play Interventions for Children with Autism at School. International Journal of Disability, Development and Education, 63(1), 45-63. https://doi.org/10.1080/1034912X.2015.1111303

Kuhaneck, H., Spitzer, S. L., \& Bodison, S. C. (2020). A Systematic Review of Interventions to Improve the Occupation of Play in Children With Autism. OTJR: Occupation, Participation and Health, 40(2), 83-98. https://doi.org/10.1177/1539449219880531

Lord, C., Risi, S., Lambrecht, L., Cook, E. H., Leventhal, B. L., DiLavore, P. C., Pickles, A., \& Rutter, M. (2000). The autism diagnostic observation schedule generic: A standard measure of social and communication deficits associated with the spectrum of autism. Journal of Autism and Developmental Disorders, 30, 205-223. https://doi.org/10.1023/A:1005592401947

Lory, C., Rispoli, M., \& Gregori, E. (2018). Play Interventions Involving Children with Autism Spectrum Disorder and Typically Developing Peers: a Review of Research Quality. Review Journal of Autism and Developmental Disorders, 5(1), 78-89. https://doi.org/10.1007/s40489-017-0124-2

Luckett, T., Bundy, A., \& Roberts, J. (2007). Do behavioural approaches teach children with autism to play or are they pretending? Autism, 11(4), 365-388. https://doi.org/10.1177/1362361307078135

Magiati, I., Tay, X. W., \& Howlin, P. (2014). Cognitive, language, social and behavioural outcomes in adults with autism spectrum disorders: A systematic review of longitudinal follow-up studies in adulthood. Clinical Psychology Review, 34(1), 78-86. https://doi.org/10.1016/j.cpr.2013.11.002

McConachie, H., Parr, J. R., Glod, M., Hanratty, J., Livingstone, N., Oono, I. P., Robalino, S., Baird, G., Beresford, B., Charman, T., Garland, D., Green, J., Gringras, P., Jones, G., Law, J., Le Couteur, A. S., Macdonald, G., McColl, E. M., Morris, C., ... Williams, K. (2015). Systematic review of tools to measure outcomes for young children with autism spectrum disorder. Health Technology Assessment, 19(41), 1-538. https://doi.org/10.3310/hta19410
Miller, L. J. (2017). Creating a common terminology for play behavior to increase cross-disciplinary research. Learning and Behavior, 45(4), 330-334. https://doi.org/10.3758/s13420017-0286-x

Munn, Z., Peters, M. D. J., Stern, C., Tufanaru, C., McArthur, A., \& Aromataris, E. (2018). Systematic review or scoping review? Guidance for authors when choosing between a systematic or scoping review approach. BMC Medical Research Methodology, 18(1). https://doi.org/10.1186/s12874-018-0611-x

Murphy, S., Joffe, V., Messer, D., Crafter, S., Radley, J., Sunthararajah, S., Bell, K., Corbacho, B., Fairhurst, C., Rodgers, S., Torgerson, D., \& Welch, C. (2019). Evaluating "enhancing pragmatic language skills for young children with social communication impairments" (E-PLAYS): Protocol for a feasibility randomised controlled trial study. Pilot and Feasibility Studies, 5(1), 75. https://doi.org/10.1186/s40814019-0456-z

O'Cathain, A., Croot, L., Duncan, E., Rousseau, N., Sworn, K., Turner, K. M., Yardley, L., \& Hoddinott, P. (2019). Guidance on how to develop complex interventions to improve health and healthcare. BMJ Open, 9(8), e029954. https://doi.org/10.1136/bmjopen-2019-029954

O'Connor, C., \& Stagnitti, K. (2011). Play, behaviour, language and social skills: $\{$ The $\}$ comparison of a play and a non-play intervention within a specialist school setting. Research in Developmental Disabilities, 32(3), 1205-1211. https://doi.org/10.1016/j.ridd.2010.12.037

Peters, M. D. J., Godfrey, C. M., Khalil, H., McInerney, P., Parker, D., \& Soares, C. B. (2015). Guidance for conducting systematic scoping reviews. International Journal of Evidence-Based Healthcare, 13(3), 141-146. https://doi.org/10.1097/XEB.0000000000000050

Quinn, S., Donnelly, S., \& Kidd, E. (2018). The relationship between symbolic play and language acquisition: A meta-analytic review. Developmental Review, 49, 121-135. https://doi.org/10.1016/j.dr.2018.05.005

Radley, K. C., Ford, W. B., Battaglia, A. A., \& McHugh, M. B. (2014). The effects of a social skills training package on social engagement of children with autism spectrum disorders in a generalized recess setting. Focus on Autism and Other Developmental Disabilities, 29(4), 216-229. https://doi.org/10.1177/1088357614525660

Rai, D., Culpin, I., Heuvelman, H., Magnusson, C. M. K., Carpenter, P., Jones, H. J., Emond, A. M., Zammit, S., Golding, J., \& Pearson, R. M. (2018). Association of autistic traits with depression from childhood to age 18 years. JAMA Psychiatry, 75(8), 835-843. https://doi.org/10.1001/jamapsychiatry.2018.1323 
Sandbank, M., Bottema-Beutel, K., Crowley, S., Cassidy, M., Feldman, J. I., Canihuante, M., \& Woynaroski, T. (2020). Intervention effects on language in children with autism: A project AIM meta-analysis. In Journal of Speech, Language, and Hearing Research (Vol. 63, Issue 5, pp. 15371560). American Speech-Language-Hearing Association. https://doi.org/10.1044/2020_JSLHR-19-00167

Skaard, G., \& Bundy, A. (2008). Test of playfulness. In L. D. Parham \& L. S. Fazio (Eds.), Play in occupational therapy for children (2nd ed., pp. 71-93). Mosby Elsevier.

Stagnitti, K., Bailey, A., Hudspeth Stevenson, E., Reynolds, E., \& Kidd, E. (2016). An investigation into the effect of play-based instruction on the development of play skills and oral language. Journal of Early Childhood Research, 14(4), 389-406. http://10.0.4.153/1476718X15579741

Strunk, J., Leisen, M., \& Schubert, C. (2017). Using a multidisciplinary approach with children diagnosed with autism spectrum disorder. In Journal of Interprofessional Education and Practice (Vol. 8, pp. 60-68). Elsevier Inc. https://doi.org/10.1016/j.xjep.2017.03.009

Thomas, J., Brunton, J., \& Graziosi, S. (2010). EPPIReviewer 4: software for research synthesis. Social Science Research Unit, UCL Institute of Education.

Thomas, J., O’Mara-Eves, A., Harden, A., \& Newman, M. (2017). Synthesis methods for combining and configuring textual or mixed methods data. In D. Gough, S. Oliver, \& J. Thomas (Eds.), An introduction to systematic reviews (pp. 181-211). Sage Publications.

Tiede, G., \& Walton, K. M. (2019). Meta-analysis of naturalistic developmental behavioral interventions for young children with autism spectrum disorder. Autism, 23(8), 2080-2095. https://doi.org/10.1177/1362361319836371

Toseeb, U., Gibson, J. L., Newbury, D. F., Orlik, W., Durkin, K., Pickles, A., \& Conti-Ramsden, G. (2020). Play and prosociality are associated with fewer externalizing problems in children with developmental language disorder: The role of early language and communication environment. International Journal of Language \& Communication Disorders, 1460-6984.12541. https://doi.org/10.1111/14606984.12541

Toth, K., Munson, J., Meltzoff, A. N., \& Dawson, G. (2006). Early predictors of communication development in young children with autism spectrum disorder: Joint attention, imitation, and toy play. Journal of Autism and Developmental Disorders, 36(8), 993-1005. https://doi.org/10.1007/s10803006-0137-7

Tricco, A. C., Lillie, E., Zarin, W., O’Brien, K. K., Colquhoun, H., Levac, D., Moher, D., Peters, M. D. J., Horsley, T., Weeks, L., Hempel, S., Akl, E. A., Chang,
C., McGowan, J., Stewart, L., Hartling, L., Aldcroft, A., Wilson, M. G., Garritty, C., ... Straus, S. E. (2018). PRISMA extension for scoping reviews (PRISMA-ScR): Checklist and explanation. Annals of Internal Medicine, 169(7), 467-473. https://doi.org/10.7326/M18-0850

Van Wijngaarden-Cremers, P. J. M., Van Eeten, E., Groen, W. B., Van Deurzen, P. A., Oosterling, I. J., \& Van Der Gaag, R. J. (2014). Gender and age differences in the core triad of impairments in autism spectrum disorders: A systematic review and meta-analysis. Journal of Autism and Developmental Disorders, 44(3), 627-635. https://doi.org/10.1007/s10803-013-1913-9

Weisberg, D. S., Hirsh-Pasek, K., \& Golinkoff, R. M. (2013). Embracing complexity: Rethinking the relation between play and learning: Comment on Lillard et al. (2013). Psychological Bulletin, 139(1), 35-39. https://doi.org/10.1037/a0030077

Weisberg, D. S., Hirsh-Pasek, K., Golinkoff, R. M., Kittredge, A. K., \& Klahr, D. (2016). Guided Play: Principles and Practices. Current Directions in Psychological Science, 25(3), 177-182. https://doi.org/10.1177/0963721416645512

Whitebread, D., Coltman, P., Jameson, H., \& Lander, R. (2009). Play, cognition and self-regulation: What exactly are children learning when they learn through play? Educational and Child Psychology, Vol.26(2), 40-52.

Wing, L., Gould, J., Yeates, S. R., \& Brierly, L. M. (1977). Symbolic play in severely mentally retarded and in autistic children. Journal of Child Psychology and Psychiatry, 18(2), 167-178. https://doi.org/10.1111/j.1469-7610.1977.tb00426.x 